\title{
Modèle de gestion d'un réservoir pour l'alimentation en eau des rizières dans un contexte de gestion intégrée des ressources en eau : cas du lac-réservoir Nui Coc au Vietnam Reservoir management model for water supply to rice fields in a context of integrated water resource management - case study: the Nui Coc lake-reservoir in Vietnam
}

\author{
Xuan Tuan Nguyen, Jean-Pierre Villeneuve, Nomessi Kokutse et Sophie \\ Duchesne
}

Volume 31, numéro 3, 2018

Reçu le 31 octobre 2017, accepté le $1^{\mathrm{er}}$ février 2018

URI : https://id.erudit.org/iderudit/1054305ar

DOI : https://doi.org/10.7202/1054305ar

Aller au sommaire du numéro

Éditeur(s)

Université du Québec - INRS-Eau, Terre et Environnement (INRS-ETE)

ISSN

1718-8598 (numérique)

Découvrir la revue

Citer cet article

Nguyen, X., Villeneuve, J.-P., Kokutse, N. \& Duchesne, S. (2018). Modèle de gestion d'un réservoir pour l'alimentation en eau des rizières dans un contexte de gestion intégrée des ressources en eau : cas du lac-réservoir Nui Coc au Vietnam. Revue des sciences de l'eau / Journal of Water Science, 31(3), 235-248. https://doi.org/10.7202/1054305ar
Résumé de l'article

Depuis plusieurs années, le Vietnam est reconnu en Asie comme un des plus gros exportateurs de riz au monde. Ce résultat a été rendu possible grâce à différents programmes du gouvernement vietnamien. Un de ces programmes a permis d'investir dans des ouvrages hydrauliques d'irrigation pour développer la production du riz. Aujourd'hui, plusieurs ouvrages hydrauliques sont construits sur le bassin versant de la rivière Cau et sont exploités à plusieurs fins. Ils servent, entre autres, les besoins en eau industrielle et domestique, à la production hydroélectrique, à l'irrigation des rizières et à la protection contre les crues. Il est donc nécessaire de définir des règles de gestion et d'opération de ces ouvrages pour satisfaire à tous les besoins en eau, et ce, particulièrement dans le cadre d'une gestion intégrée des ressources en eau à l'échelle du bassin versant. L'objectif du travail présenté dans cet article est de modéliser l'évolution des volumes d'eau d'un réservoir et de proposer une méthode pour gérer un réservoir utilisé à plusieurs fins, dont l'irrigation des rizières. Un cas particulier est présenté pour le lac-réservoir Nui Coc, au Vietnam. Dans le cadre d'un projet de gestion intégrée des ressources en eau du bassin versant de la rivière Cau, sur lequel le lac-réservoir Nui Coc est situé, le modèle Hydrotel a été utilisé pour les simulations hydrologiques. Or ce modèle ne possédait pas de sous-modèle de gestion de réservoir adapté à l'irrigation des rizières ni à la prise en compte de divers types de besoins en eau. Il a donc fallu développer un sous-modèle comprenant deux modules et l'adapter à la problématique d'irrigation des rizières à partir du lac Nui $\mathrm{Coc}$. Ce sous-modèle ainsi développé a permis de reproduire, avec le modèle Hydrotel ainsi modifié, la variation historique du volume d'eau dans le lac Nui Coc puis d'évaluer les possibilités d'expansion des rizières. 


\section{MODĖLE DE GESTION D’UN RÉSERVOIR POUR}

\section{L'ALIMENTATION EN EAU DES RIZIĖRES DANS UN CONTEXTE \\ DE GESTION INTÉGRÉE DES RESSOURCES EN EAU : CAS DU LAC-RÉSERVOIR NUI COC AU VIETNAM}

Reservoir management model for water supply to rice fields in a context of integrated water resource management - case study:

the Nui Coc lake-reservoir in Vietnam

Xuan Tuan NGUyEN, Jean-PIerre VILleneuVE, Nomessi KOKUTSE, Sophie DUCHESNE**

Centre Eau Terre Environnement, Institut national de la recherche scientifique (INRS), 490, rue de la Couronne, Québec

(Québec) G1K 9A9, Canada

Reçu le 31 octobre 2017, accepté le $1^{\text {er }}$ février 2018

\section{RÉSUMÉ}

Depuis plusieurs années, le Vietnam est reconnu en Asie comme un des plus gros exportateurs de riz au monde. Ce résultat a été rendu possible grâce à différents programmes $\mathrm{du}$ gouvernement vietnamien. Un de ces programmes a permis d'investir dans des ouvrages hydrauliques d'irrigation pour développer la production du riz. Aujourd'hui, plusieurs ouvrages hydrauliques sont construits sur le bassin versant de la rivière Cau et sont exploités à plusieurs fins. Ils servent, entre autres, les besoins en eau industrielle et domestique, à la production hydroélectrique, à l'irrigation des rizières et à la protection contre les crues. Il est donc nécessaire de définir des règles de gestion et d'opération de ces ouvrages pour satisfaire à tous les besoins en eau, et ce, particulièrement dans le cadre d'une gestion intégrée des ressources en eau à l'échelle du bassin versant. L'objectif du travail présenté dans cet article est de modéliser l'évolution des volumes d'eau d'un réservoir et de proposer une méthode pour gérer un réservoir utilisé à plusieurs fins, dont l'irrigation des rizières. Un cas particulier est présenté pour le lac-réservoir Nui Coc, au Vietnam. Dans le cadre d'un projet de gestion intégrée des ressources en eau du bassin versant de la rivière Cau, sur lequel le lac-réservoir Nui Coc est situé, le modèle Hydrotel a été utilisé pour les simulations hydrologiques. Or ce modèle ne possédait pas de sous-modèle de gestion de réservoir adapté à l'irrigation des rizières ni à la prise en compte de divers types de besoins en eau. Il a donc fallu développer un sous-modèle comprenant deux modules et l'adapter à la problématique d'irrigation des rizières à partir du lac Nui Coc. Ce sous-modèle ainsi développé a permis de reproduire, avec le modèle Hydrotel ainsi modifié, la variation historique du volume d'eau dans le lac Nui Coc puis d'évaluer les possibilités d'expansion des rizières.

Mots-clés : modèle hydrologique, modèle des rizières, irrigation, bilan hydrique, gestion du lac, gestion intégrée des ressources en eau. 


\section{ABSTRACT}

For several years, Vietnam has been recognized in Asia as one of the largest rice exporting countries in the world. This result was made possible through various programs of the Vietnamese government. One of these programs was to invest in irrigation hydraulic infrastructures to develop rice production. Today, several hydraulic infrastructures are built on the catchment of the Cau River and are exploited for several purposes. They serve, among others, industrial and domestic water supply, hydroelectric production, irrigation of rice fields and protection against floods. It is therefore necessary to define rules for the management and operation of these structures in order to satisfy all water needs, especially in the context of integrated water resources management at the watershed scale. The objective of this paper is to model the evolution of the water volumes of a reservoir and to propose a method for managing a reservoir used for several purposes, including irrigation of rice fields. A special case is presented for the Nui Coc lake-reservoir in Vietnam. As part of an integrated water resource management project for the Cau River watershed, on which the Nui Coc reservoir is located, the Hydrotel model was used for hydrological simulations. However, this model did not have a reservoir management sub-model suitable for irrigating rice fields and taking into account various types of water requirements. It was therefore necessary to develop a submodel including two modules and to adapt it to the problem of irrigation of rice fields from Lake Nui Coc. This sub-model allowed reproducing, with the Hydrotel model modified, the historical variation of the volume of water in Lake Nui Coc then to evaluate the possibilities of expansion of the rice fields.

Key words: hydrological model, rice fields modeling, irrigation, water balance, lake management, integrated water resources management.

\section{INTRODUCTION}

Dans le cadre du projet de gestion intégrée des ressources en eau du bassin versant de la rivière Cau du Vietnam, il a été convenu que le modèle GIBSI, développé à l'Institut National de la Recherche Scientifique (INRS) (VILLENEUVE et al., 1998; VILLENEUVE et al., 2003; ROUSSEAU et al., 2000) serait mis en place pour évaluer différents scénarios de gestion.

Dans l'outil informatique de gestion intégrée GIBSI, Hydrotel (FORTIN et al., 1995) est le modèle utilisé pour modéliser et simuler l'ensemble des processus hydrologiques d'un bassin versant. Ce modèle permet de tenir compte des aménagements et des ouvrages hydrauliques (infrastructures).
Le bassin versant de la rivière Cau (Figure 1) présente une problématique intéressante en ce qui a trait à l'alimentation en eau des rizières et à la gestion des réservoirs servant à cette alimentation. Or dans le modèle Hydrotel, bien qu'il existe des fonctions permettant de gérer des lacs, des barrages ou des prises d'eau, il n'y a pas de sous-modèle d'irrigation des rizières ni de sous-modèle de gestion de réservoirs adapté à l'irrigation. NGUYEN (2016) et NGUYEN et al. (2018) ont développé un modèle d'irrigation des rizières qui a été intégré à Hydrotel. Dans ce contexte, le premier objectif du présent article est de développer le sous-modèle de gestion du réservoir, de le valider, de l'intégrer à Hydrotel puis d'appliquer Hydrotel ainsi modifié à la gestion d'un réservoir d'alimentation pour l'irrigation des rizières. Le deuxième objectif est de vérifier la capacité du lacréservoir Nui Coc à répondre aux besoins en irrigation et de voir s'il y a possibilité de développement des rizières dans ce bassin versant.

\section{PROBLÉMATIQUES}

\subsection{Bassin versant de la rivière Cong}

Dans le bassin versant de la rivière Cong, la culture la plus importante est la culture du riz, laquelle demande d'abondantes quantités d'eau. Pour satisfaire ces besoins en eau, un réservoir artificiel (lac-réservoir Nui Coc) et un système de canaux d'irrigation ont été construits entre 1973 et 1976 (MRE, 1988). Le réservoir, dont le volume utile a été fixé à $167,9 \times 10^{6} \mathrm{~m}^{3}$ lors de sa conception (OGINK et al., 2005), a été mis en service en 1977 . L'objectif principal de cet ouvrage était, à l'origine, l'irrigation des rizières et l'alimentation en eau pour les usages industriels et domestiques.

Le lac est maintenant utilisé à différentes fins et particulièrement pour l'irrigation (Figure 2). Il sert à l'alimentation industrielle et domestique pendant toute l'année. En saison des crues, il est utilisé pour la régulation des débits. En saison sèche, il sert à l'irrigation des rizières situées tant à l'extérieur qu'à l'intérieur du bassin versant. Le lac alimente également le réservoir du barrage Thac Huong, situé à l'extérieur du bassin versant. Aujourd'hui, on constate une augmentation de la surface des rizières et donc un accroissement important des besoins en eau. La figure 2 présente une illustration schématisée des différents débits entrant et sortant du lac.

La mise en place de cet ouvrage-réservoir a grandement modifié l'écoulement de la rivière par rapport à l'écoulement naturel. On peut croire, a priori, qu'actuellement les ressources en eau dans ce bassin versant sont exploitées au maximum. De plus, la répartition de la ressource entre les différents consommateurs crée des conflits. En raison de ces différentes 


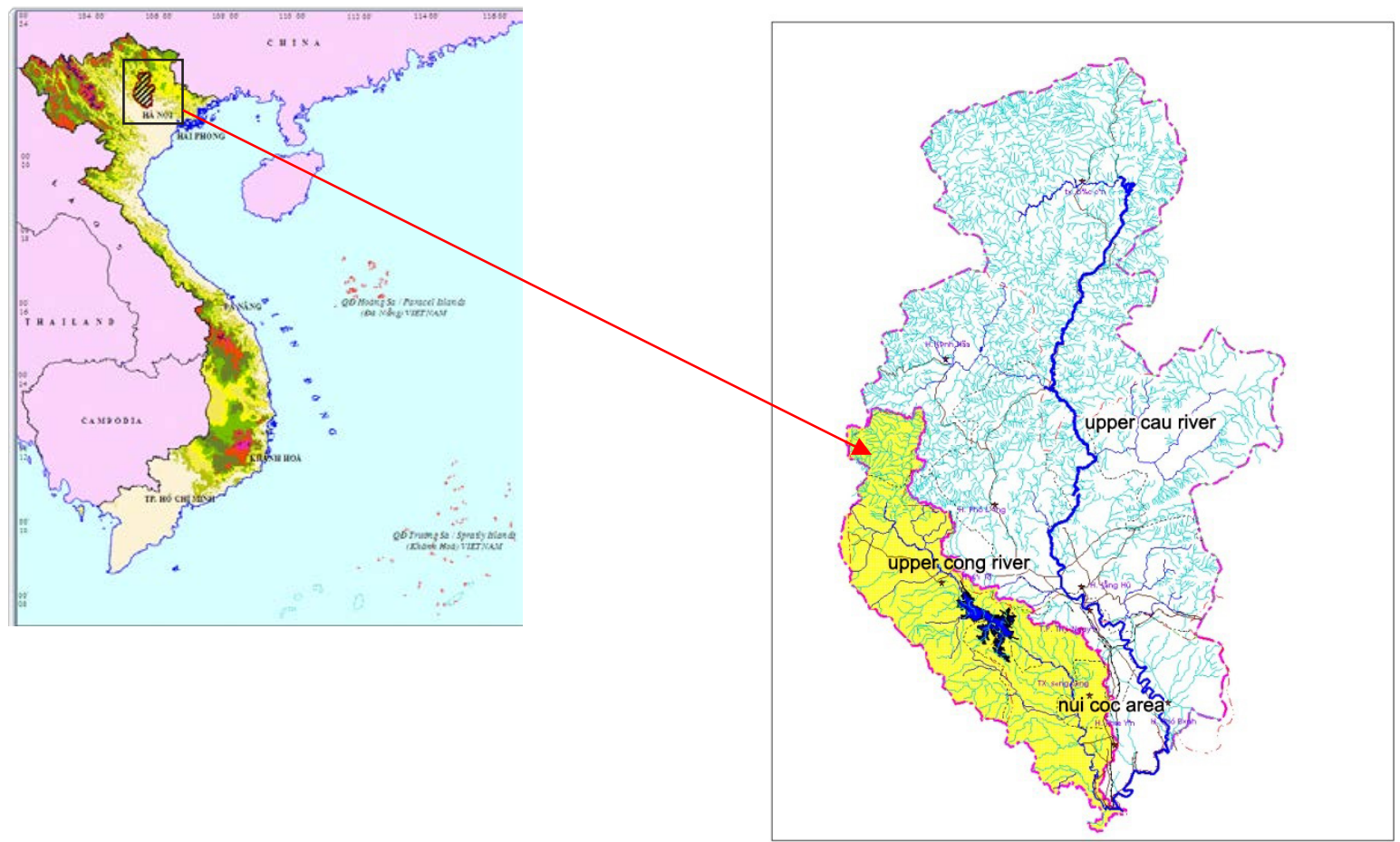

Figure 1. Localisation des bassins versants des rivières Cau et Cong au Vietnam. Location of the watersheds of the Cau and Cong rivers in Vietnam.

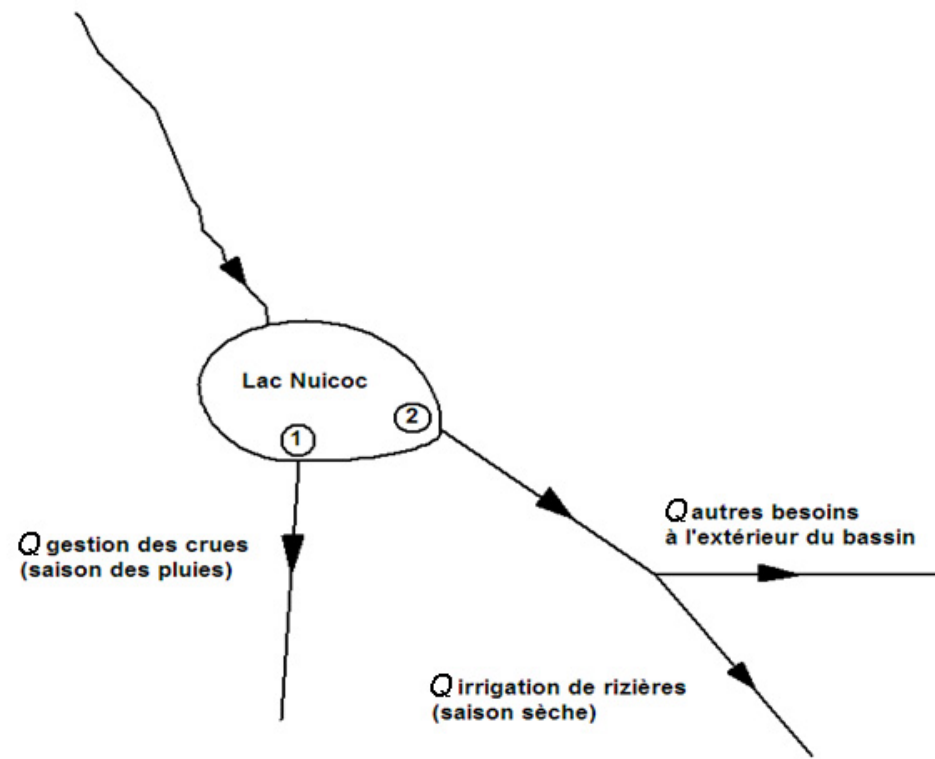

Figure 2. Schéma simple d'opération du lac Nui Coc. $Q$ : débits; (1) : déversoir; (2) : sortie pour l'irrigation et les autres besoins. Simplified scheme of Nui Coc Lake operation. Q: flows (1): weir; (2) output for irrigation and other needs.

demandes, la demande en eau pour les rizières n'est pas toujours garantie, ce qui a pour effet de réduire le rendement de la production en riz. Il s'avère donc nécessaire d'évaluer la gestion du lac-réservoir en raison de son impact sur le développement rizicole.

\subsection{Nécessité de développer des sous-modèles}

Dans le modèle Hydrotel (FORTIN et al., 1995), il n'existait pas de sous-modèle d'irrigation des rizières. Le sousmodèle-rizière développé et validé par NGUYEN (2016) et NGUYEN et al. (2018) a été intégré au modèle Hydrotel.

Dans le modèle Hydrotel, il existe des fonctions permettant de gérer des lacs, des barrages ou des prises d'eau. Cependant, ces fonctions ne sont pas adaptées à la problématique de l'irrigation ni à la gestion de multiples demandes, telles que celles présentes dans la problématique de gestion du lac Nui Coc. Pour réaliser la gestion du lac, il faut donc développer des sous-modèles pour pouvoir gérer ce cas particulier. Ce sont ces sous-modèles qui seront utilisés dans Hydrotel pour cette problématique d'irrigation.

\subsection{Schéma général d'opération des sous-modèles dans Hydrotel}

Sur la base de la structure des sous-modèles dans Hydrotel, on présente (Figure 3), l'organigramme du nouveau modèle tenant compte du sous-modèle de gestion des rizières et du sous-modèle de gestion du lac. Cet organigramme, tiré de NGUYEN (2016) et NGUYEN et al. (2018), permet de visualiser les liens entre les données et les modèles. 


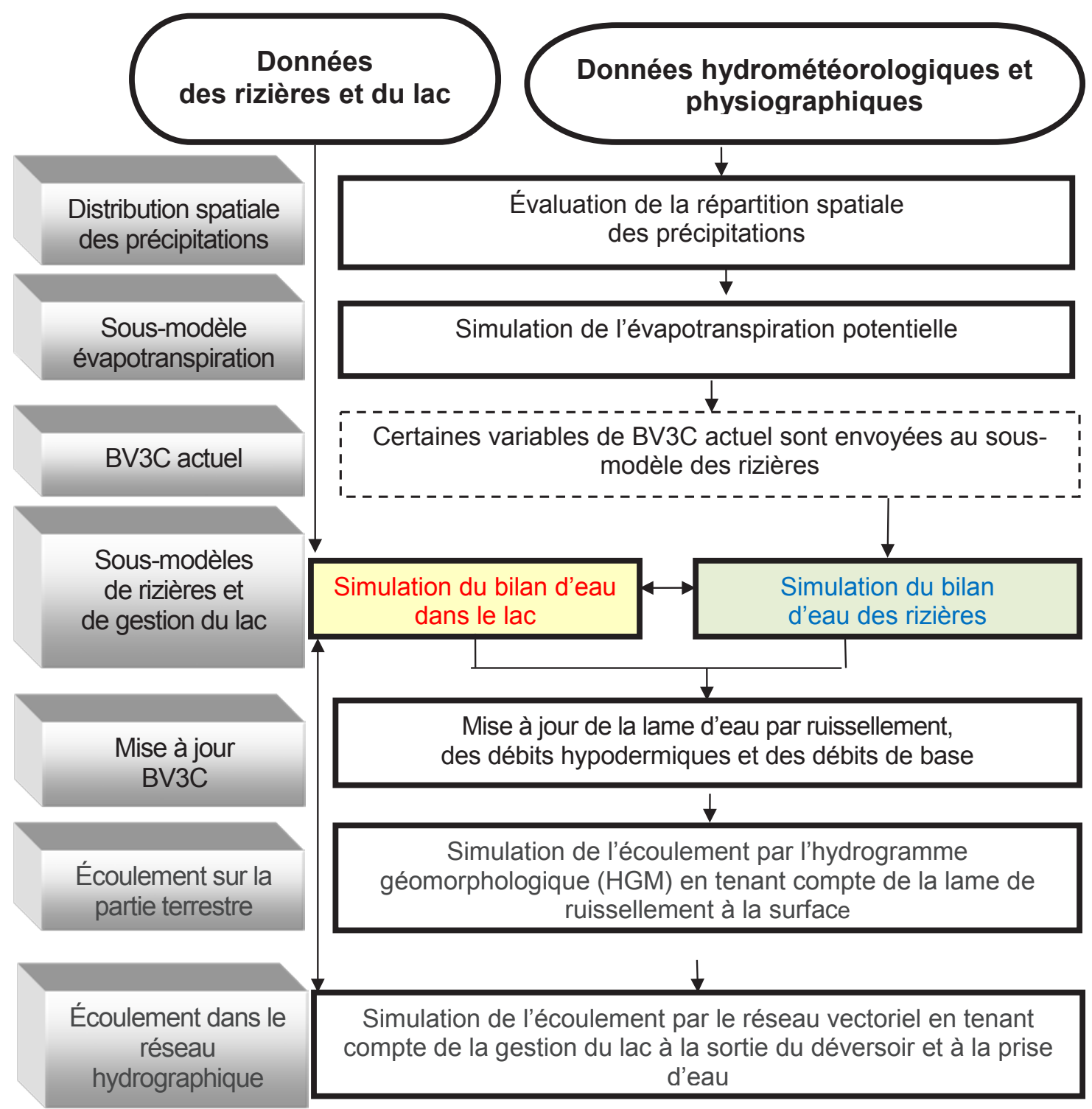

Figure 3. Schéma du modèle Hydrotel modifié (les deux sous-modèles ajoutés au modèle Hydrotel sont en couleur).

Diagram of the modified Hydrotel model (the two sub-models added to Hydrotel are in color).

\subsection{Liens du sous-modèle de gestion du lac-réservoir avec Hydrotel}

Pour modéliser la gestion du lac, on n'utilise pas la modélisation de lac telle que réalisée par Hydrotel. On établit un sous-modèle de gestion applicable aux problématiques de gestion du lac Nui Coc. Ce sous-modèle sera utilisé, dans le schéma d'écoulement modifié d'Hydrotel, en remplacement $\mathrm{du}$ module original de gestion des lacs d'Hydrotel. Pour mettre en place ce sous-modèle, on doit tenir compte : (i) de la géométrie du lac, de son volume utile, de son volume mort et de la capacité de transport du canal principal et (ii) de l'ensemble des demandes en eau, soit celles pour maintenir un débit minimal en rivière, pour la production d'électricité, pour l'industrie, pour le barrage Thac Huong et pour l'irrigation des rizières à l'extérieur et à l'intérieur du bassin versant. On doit aussi prendre en compte les pertes en eau dans les canaux d'irrigation.

La gestion du lac est effectuée à chaque pas de temps de simulation d'Hydrotel. On calcule d'abord l'ensemble des besoins en irrigation à l'aide du sous-modèle de rizières. Ensuite, on détermine les différentes consommations ainsi que les pertes en eau des canaux d'irrigation. Dans la procédure de gestion du lac, la quantité d'eau disponible pour l'irrigation est calculée en fonction de la capacité maximale de transport du canal principal et des autres besoins en eau. La quantité d'eau nécessaire à satisfaire l'ensemble des besoins est alors retirée du lac et on établit le bilan en eau du lac. 
Le schéma représentant le sous-modèle permettant la gestion du lac, adapté et implémenté en tenant compte de la structure du modèle Hydrotel et de son fonctionnement, est présenté à la figure 4 .

L'équation de bilan d'eau dans le lac s'écrit de la façon suivante pour chaque pas de temps $i$ :

$$
V_{l a c}^{i}=V_{l a c}^{i-1}+\left(V_{e}^{i}-V_{p}^{i}\right)-V_{s}^{i}-V_{c s}^{i}
$$

où $V_{l a c}^{i-1}$ : volume d'eau du lac à la fin du jour $i-1\left(\mathrm{~m}^{3}\right)$;

$V_{l a c}^{i}$ : volume d'eau du lac au jour $i\left(\mathrm{~m}^{3}\right)$;

$V_{p}^{i}$ : volume des pertes par évaporation et par infiltration d'eau du lac au jour $i\left(\mathrm{~m}^{3}\right)$; les pertes par infiltration étant considérées comme négligeables, ce terme n'exprime que :

$$
V_{p}^{i}=\mathrm{ET}^{i} \times S_{l a c}^{i}
$$

où $\mathrm{ET}_{i}$ : perte au jour $i$ par évaporation dans le lac (m), qui est calculée par l'algorithme d'Hydrotel (voir FORTIN et ROYER, 2004);

$S_{\text {lac }}^{i}$ : surface du lac au jour $i$, déterminée par l'équation de la relation surface $v$ s élévation du lac $\left(\mathrm{m}^{2}\right)$;

$V_{e}^{i}$ : quantité d'eau pendant le jour $i$ provenant du tronçon en amont du lac et des unités de simulation (UHRH) avoisinant le lac $\left(\mathrm{m}^{3}\right)$;

$$
V_{e}^{i}=\left(Q_{a p}^{i}+\mathrm{Q}_{1}^{\mathrm{i}}+Q_{2}^{i}\right) \times \Delta \mathrm{t}
$$

où $Q_{a p}$ : débit apporté par le tronçon de rivière en amont du lac $\left(\mathrm{m}^{3} \cdot \mathrm{s}^{-1}\right)$ (Figure 4);

$Q_{1}$ : débit de ruissellement qui arrive directement au lac $\left(\mathrm{m}^{3} \cdot \mathrm{s}^{-1}\right)$ (Figure 4);

$Q_{2}$ : débit provenant de l'écoulement hypodermique $\left(\mathrm{m}^{3} \cdot \mathrm{s}^{-1}\right)$ (Figure 4);

$\Delta t:$ pas de temps (s).

$V_{s}^{i}$ : quantité d'eau sortant vers le tronçon en aval du lac le jour $i\left(\mathrm{~m}^{3}\right)$;

$V_{c s}^{i}:$ quantité d'eau prélevée du réservoir le jour $i$ pour répondre aux trois besoins de consommations :

$$
V_{c s}^{i}=\left(1+K_{p}\right)\left(V_{i n d}^{i}+V_{b r}^{i}+V_{r i z}^{i}\right)
$$

où $K_{p}$ : coefficient moyen journalier de pertes d'eau dans les canaux d'irrigation. Ce coefficient permet de tenir compte des pertes par évaporation, par infiltration, par débordement, etc.;

$V_{\text {ind }}^{i}$ : quantité d'eau prélevée le jour $i$ pour répondre aux besoins des industries $\left(\mathrm{m}^{3}\right)$;

$V_{b r}^{i}$ : quantité d'eau prélevée le jour $i$ pour alimenter le barrage Thac Huong $\left(\mathrm{m}^{3}\right)$;
$V_{r i z}^{i}$ : quantité d'eau prélevée le jour $i$ pour l'irrigation des rizières intérieures et extérieures au bassin versant à l'étude. Cette valeur est calculée comme suit :

$$
V_{r i,}^{i}=V_{r_{-} i}^{i}+V_{r_{-} e}^{i}
$$

où $V_{r_{-} i}^{i}$ : volume d'eau nécessaire pour l'irrigation des rizières à l'intérieur du bassin versant calculé par le sousmodèle des rizières, voir NGUYEN (2016) et NGUYEN et al. (2018);

$V_{r_{-}}^{i}$ : volume d'eau nécessaire pour l'irrigation des rizières à l'extérieur du bassin versant.

Le sous-modèle développé par NGUYEN (2016) et NGUYEN et al. (2018), pour tenir compte des besoins en eau des rizières, calcule les besoins en eau seulement pour les rizières qui sont à l'intérieur du bassin versant. Pour déterminer la quantité d'eau d'irrigation nécessaire pour alimenter les rizières extérieures au bassin versant, on fait les hypothèses suivantes : (i) les rizières à l'extérieur du bassin sont soumises aux mêmes conditions climatiques que celles à l'intérieur du bassin; (ii) les conditions de sol et la topographie sont aussi sensiblement les mêmes et (iii) les règles de gestion des cultures et les besoins en eau sont identiques à ceux des parcelles à l'intérieur du bassin. Ainsi, pour la gestion du réservoir, les besoins en eau des parcelles à l'extérieur du bassin versant qui sont irriguées par le même réseau de canaux d'irrigation (et non pas par le barrage Thac Huong) seront considérés comme identiques, par unité de surface, à ceux des parcelles à l'intérieur du bassin. On utilisera alors un coefficient des besoins défini de la façon suivante :

$$
K_{c}^{r}=\frac{S_{e}^{r}}{S_{i}^{r}}
$$

où $K_{c}^{r}$ : proportion de la surface des rizières extérieures par rapport à celles intérieures au bassin versant;

$S_{e}^{r}$ : surface des rizières à l'extérieur du bassin versant (ha);

$S_{i}^{r}$ : surface des rizières à l'intérieur du bassin versant (ha).

Les contraintes de gestions du réservoir sont définies à partir des contraintes établies lors de la mise en exploitation du réservoir. Elles sont illustrées dans le schéma représentant le réservoir à la figure 5 .

La quantité d'eau sortant du réservoir vers le tronçon de rivière en aval du lac au jour $i, V_{s}^{i}$, est fixée à un minimum de $0,168 \mathrm{~m}^{3} \cdot \mathrm{s}^{-1}$. Ce débit est requis pour satisfaire en tout temps la demande écologique de la rivière. Lorsque le niveau du réservoir est plus haut que la cote maximale permise, on fait l'hypothèse que toute l'eau excédentaire est déversée à la rivière par le déversoir. 


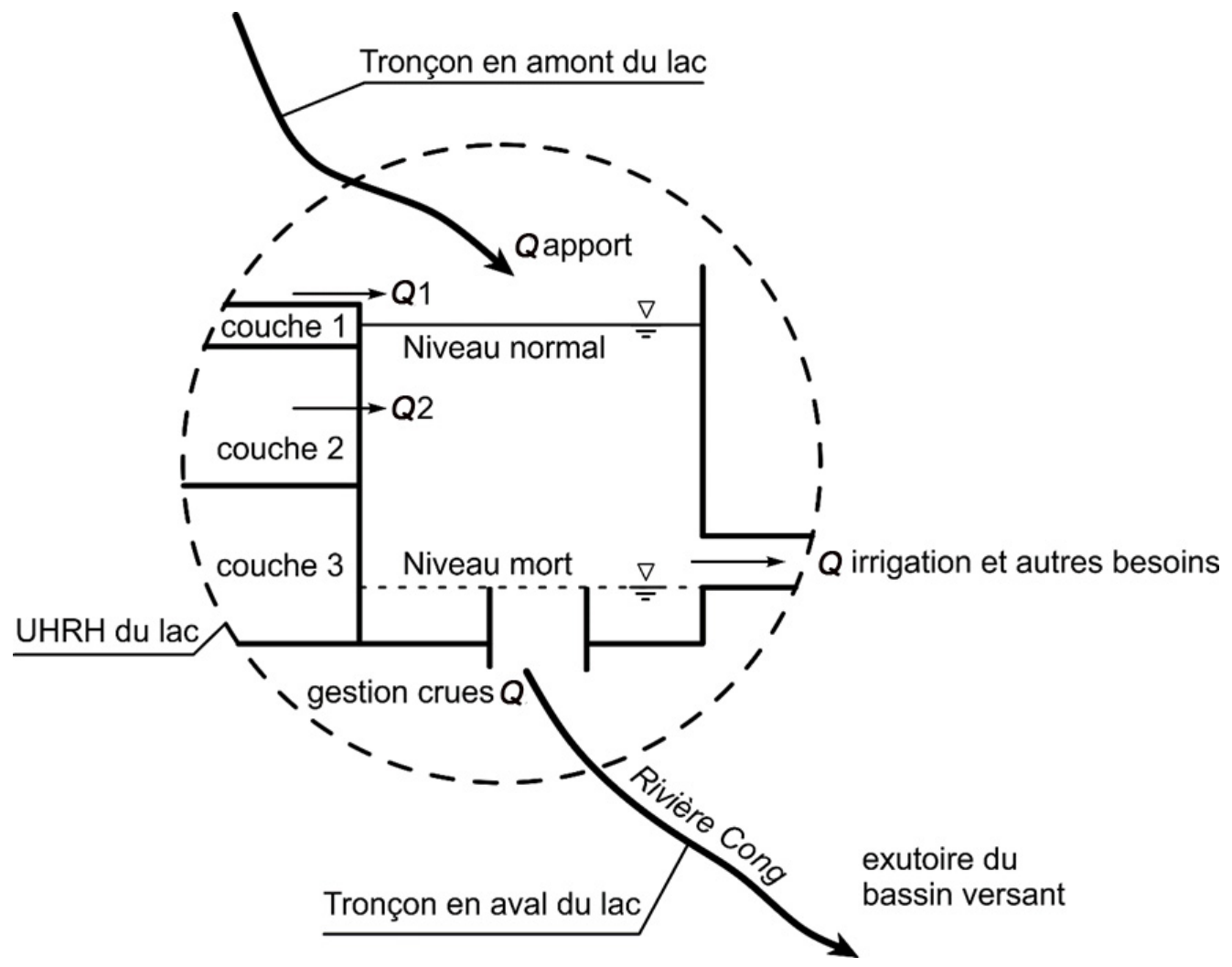

Figure 4. Illustration de la gestion du lac dans Hydrotel. $Q_{1}$ : débit de ruissellement arrivant au lac; $Q_{2}$ : débit provenant de l'écoulement hypodermique; couches 1,2 et 3 selon le concept de découpage en couches d'Hydrotel.

Illustration of $H y d r o t e l$ lake management module. $Q_{1}$ : runoff flow to the lake; $Q_{2}$ : flow from hypodermic flow; layers 1, 2 and 3 according to Hydrotel's layered concept.

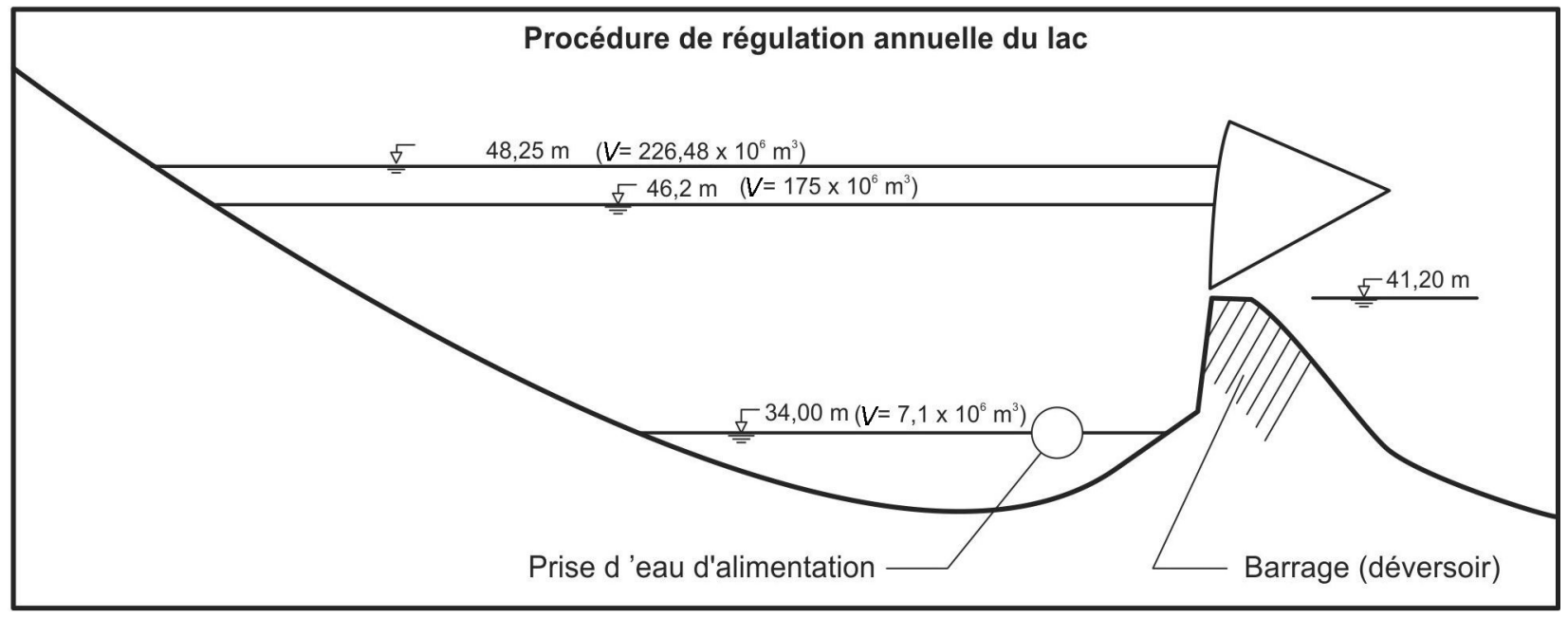

Figure 5. Illustration des contraintes d'opération du lac-réservoir Nui Coc. Illustration of the operating constraints for the Nui Coc reservoir. 
Les contraintes d'opération liées au critère de design du lac imposent des niveaux minimal et maximal de gestion. Ainsi, pour le seuil maximal, la contrainte est $H_{\text {lac }} \leq 46,2 \mathrm{~m}$ où $H_{\text {lac }}$ est la hauteur d'eau du lac (m). Exceptionnellement, le niveau d'eau peut atteindre $48,25 \mathrm{~m}$ dans un contexte de gestion de crues exceptionnelles. Le niveau minimal doit respecter la contrainte suivante : $H_{\text {lac }} \geq 34,0 \mathrm{~m}$.

L'organigramme du sous-modèle de gestion du réservoir est donné à la figure 6 . Cet organigramme décrit la séquence des opérations de gestion qui sont ajoutées au modèle Hydrotel. On retrouve à la figure 3, l'organigramme décrivant la structure d'opération d'Hydrotel. On notera dans cette figure où a été implanté le modèle de gestion du réservoir dans la structure d'Hydrotel.

\subsection{Calage des différents modèles}

Suite à la construction et à la mise en service du réservoir Nui Coc, la dernière station hydrologique située en aval du lac qui était alors encore en service dans le bassin versant, Tan Cuong, a été fermée. Aucune donnée observée de débits en rivière n’est donc disponible pour le calage des sous-modèles de rizières selon la méthode traditionnelle (simulation et comparaison des débits simulés avec les débits observés). Ainsi, certaines hypothèses ont dû être considérées. Tout d'abord, on sait que la riziculture est également pratiquée dans d'autres sous-bassins versants de la rivière Cau en saison des pluies et on considère que les caractéristiques (pluviométrie, température, types de sols, etc.) de ces sous-bassins versants peuvent être considérées comme similaires à celles du bassin versant de la rivière Cong. La riziculture pratiquée dans la partie amont du bassin versant de la rivière Cau suit les mêmes règles que celle pratiquée dans le bassin versant de la rivière Cong (dates de culture, hauteur de drainage des casiers rizicoles, etc.). Par conséquent, en saison des pluies, le calage du sous-modèle de rizières a été réalisé à partir des débits mesurés à l'exutoire de la partie amont du bassin versant de la rivière Cau. Les valeurs de paramètres obtenues lors de ce calage ont ensuite été utilisées pour le bassin versant de la rivière Cong. Dans le bassin versant de la rivière Cau, des données observées sont disponibles à la station Gia Bay pour la période d'étude retenue; elles seront donc utilisées pour le calage des paramètres d'Hydrotel modifié. La figure 7 indique la position de la station Gia Bay sur le bassin versant de la rivière Cau ainsi que la localisation du lac-réservoir Nui Coc au sud-ouest de ce bassin versant.

\subsubsection{Paramètres de calage}

Pour la valeur des paramètres du modèle Hydrotel, on utilise, dans un premier temps, les paramètres de calage qui ont été obtenus par NGUYEN (2012) pour le modèle Hydrotel non modifié. Ensuite, on cale les paramètres du modèle de rizières, soit le coefficient de culture du riz et le coefficient d'infiltration. Enfin, on cale le paramètre de perte par les canaux d'irrigation, qui intervient directement dans le sousmodèle de gestion du lac.

Les paramètres du modèle de rizières ont été calés par NGUYEN (2016) et NGUYEN et al. (2018) en utilisant les données disponibles de débits observés à la station Gia Bay. Les débits journaliers des années 2006 et 2007 ont été utilisés pour le calage et ceux des années 2008 et 2009 pour la validation. La comparaison des débits observés et simulés présentée à la figure 8 , montre que le calage a permis de bien représenter les débits observés de façon générale. La comparaison des volumes mensuels, présentée à la figure 9, indique aussi que la valeur des paramètres obtenue lors du calage permet de bien représenter les volumes mensuels observés.

En utilisant les valeurs des paramètres obtenus pour Hydrotel par NGUYEN (2012) et pour le sous-modèle de rizières par NGUYEN (2016), le seul paramètre dont la valeur n'est pas encore déterminée est celle du coefficient moyen journalier de pertes d'eau dans les canaux d'irrigation, $K_{p}$. Cette valeur est obtenue en tenant compte du bilan des volumes utiles du lac et des consommations prises dans le lac pour une année de référence donnée. Lors de la mise en place du réservoir, la valeur théorique pour $K_{p}$ a été fixée à 0,30 . C'est cette valeur qui a été utilisée comme valeur initiale pour le calage. Après plusieurs simulations, la valeur de 0,423 est celle qui permettait d'obtenir la meilleure adéquation entre le bilan du lac et les demandes en eau en utilisant la pluviométrie de l'année 1966. La pluviométrie annuelle de 1966 a une probabilité de dépassement de $75 \%$, selon les données de 1961-1972, et elle correspond à celle utilisée pour définir les critères de design du réservoir.

\section{RÉSULTATS}

En utilisant Hydrotel incluant les deux nouveaux sousmodèles, l'évolution du volume d'eau dans le lac Nui Coc a été simulée pour l'année 1988, qui est considérée comme une année sèche puisque la pluviométrie totale au cours de cette année correspond à une probabilité de dépassement de $85 \%$.

La figure 10 présente, à titre d'exemple, les courbes cumulées des différents besoins en eau simulés pour l'année 1988. Au début de l'année, le volume total d'eau dans le lac est de $167,9 \times 10^{6} \mathrm{~m}^{3}$ (Figure 11). Pour l'année 1988, le sousmodèle de rizières d'Hydrotel calcule un volume total d'eau nécessaire pour l'irrigation des rizières à l'intérieur du bassin versant de $20,3 \times 10^{6} \mathrm{~m}^{3}(13 \%)$. Pour les besoins en irrigation à l'extérieur du bassin, le volume nécessaire est donc évalué à $40,4 \times 10^{6} \mathrm{~m}^{3}(26 \%)$. Les besoins pour l'industrie ont été évalués 


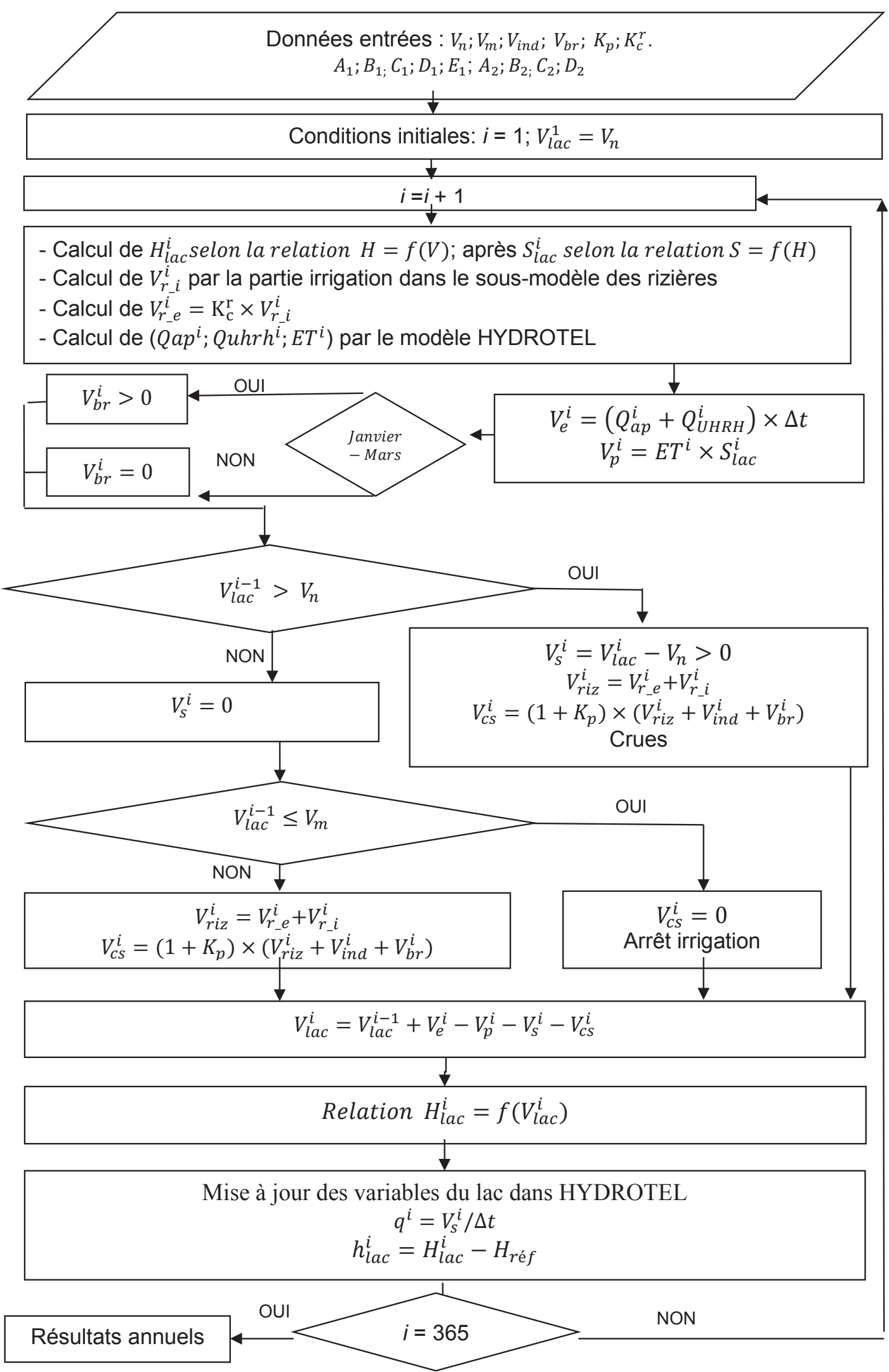

Figure 6. Organigramme de gestion des volumes du réservoir. Management flow chart of the reservoir volumes. 


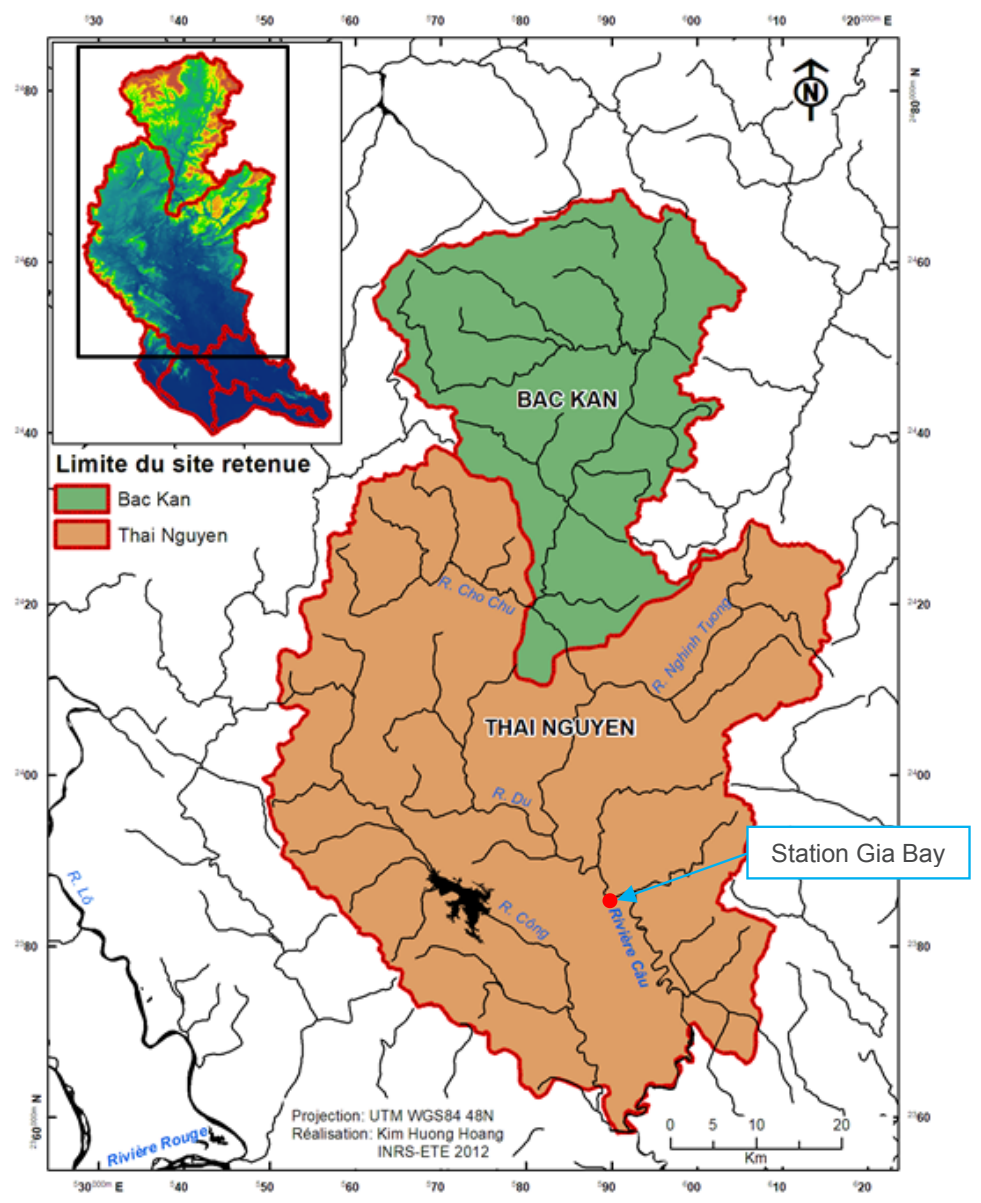

Figure 7. Localisation de la station Gia Bay sur le bassin versant de la rivière Cau (le lac-réservoir Nui Coc est visible au sud-ouest). Location of Gia Bay station in the Cau River watershed (Nui Coc Lake-reservoir is visible at the southwest).

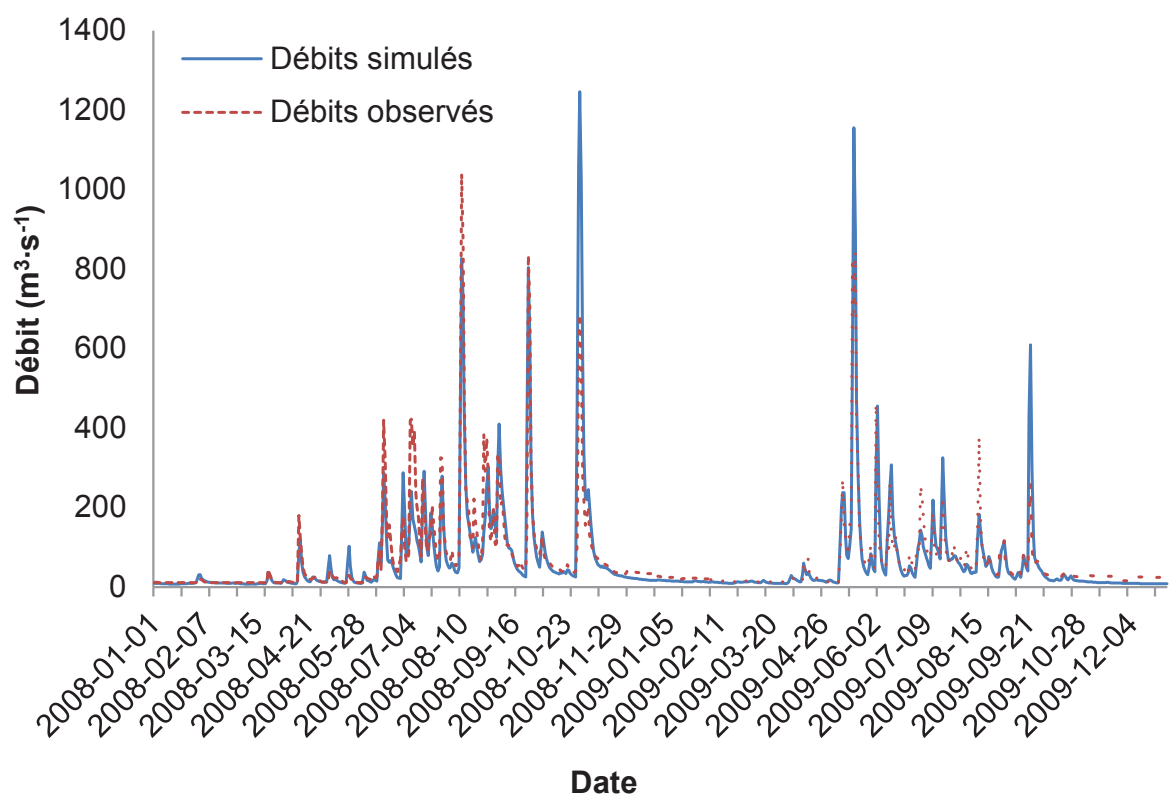

Figure 8. Débits observés et simulés à la station Gia Bay pour la période 2008-2009. Observed and simulated flows at Gia Bay station for the period 2008-2009. 


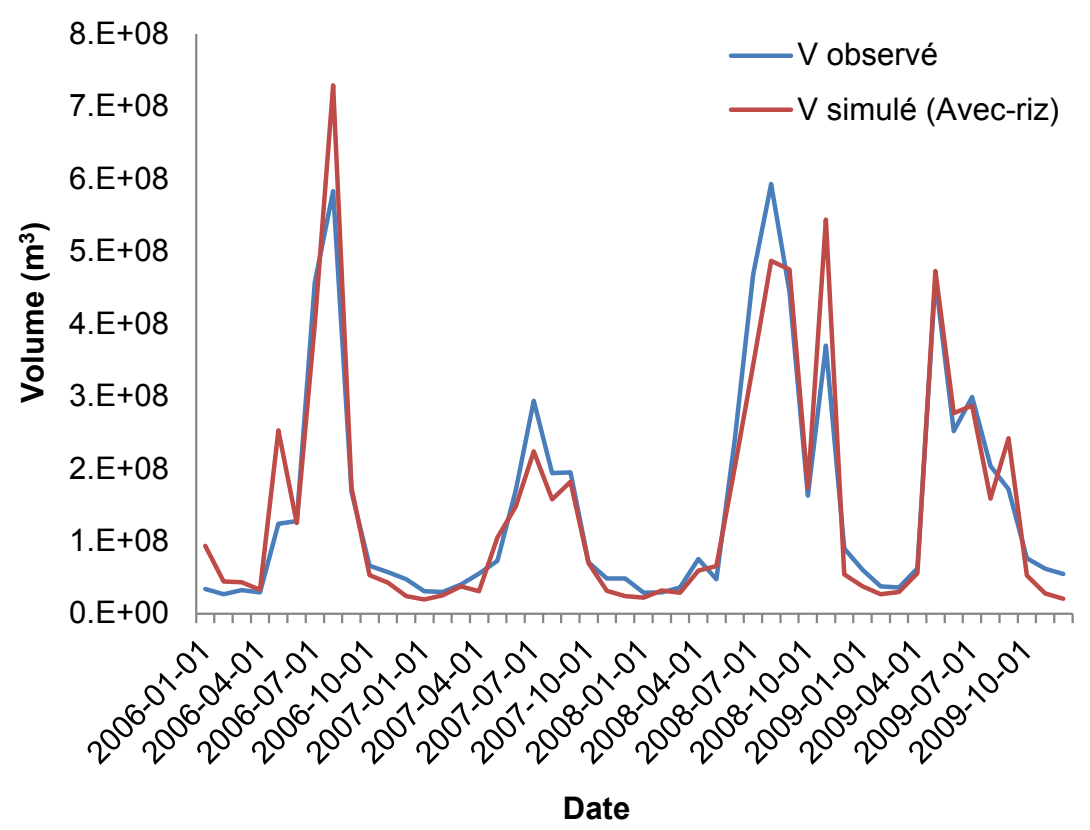

Figure 9. Volume d'eau mensuel à la station Gia Bay pendant la période 2006-2009. Monthly water volume at Gia Bay station for the 2006-2009 period.

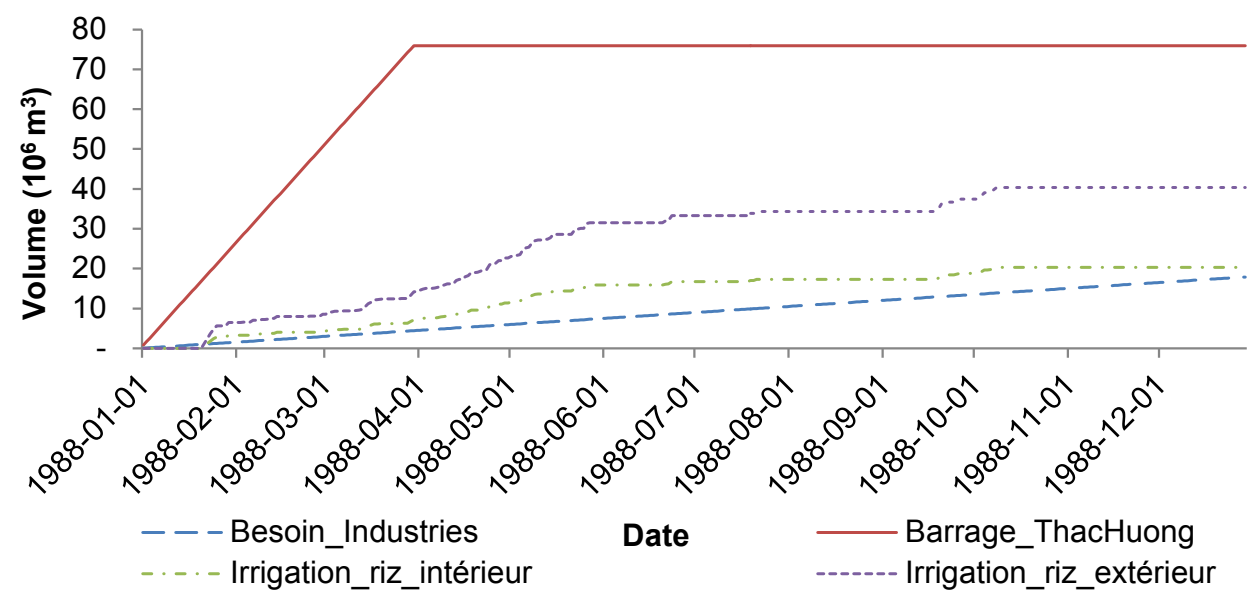

Figure 10. Volumes cumulés des consommations prises dans le lac en 1988. Cumulative consumption volumes taken from the lake in 1988.

à $17,9 \times 10^{6} \mathrm{~m}^{3}(12 \%)$ et les besoins pour l'alimentation $\mathrm{du}$ barrage Thac Huong sont évalués à $75,9 \times 10^{6} \mathrm{~m}^{3}(49 \%)$ (LE, 2011). La figure 12 présente les volumes cumulés entrant et sortant du lac pour l'année 1988. On constate que, pour cette année, le total des apports au lac est de $336,9 \times 10^{6} \mathrm{~m}^{3}$ et que le total des besoins en eau est de $154,47 \times 10^{6} \mathrm{~m}^{3}$. Le volume d'eau dans le lac en début d'année étant de $167,9 \times 10^{6} \mathrm{~m}^{3}$, le volume résiduel dans le lac en fin d'année, sans tenir compte des apports qui ne surviennent majoritairement que dans la deuxième moitié de l'année, est donc de $13,53 \times 10^{6} \mathrm{~m}^{3}$. Le niveau du lac n'a pas atteint le niveau du volume mort. À la figure 11 , on présente l'évolution du volume d'eau dans le lac selon les résultats de simulation. On constate que l'eau commence à s'accumuler dans le lac à partir du 21-06-1988 pour atteindre un volume maximal de $160 \times 10^{6} \mathrm{~m}^{3}$ le $01-11$ 1988. On doit souligner que ce volume maximal est plus petit que la capacité maximale du lac, qui est de $175 \times 10^{6} \mathrm{~m}^{3}$. Le fait de ne pas avoir atteint la capacité maximale du lac pourrait présenter des difficultés à satisfaire l'irrigation dans l'année suivante.

\subsection{Analyse de la capacité du lac à répondre aux besoins en irrigation des rizières}

Pour analyser la capacité du lac à répondre aux besoins d'irrigation des rizières, l'évolution du volume d'eau du lac a été 


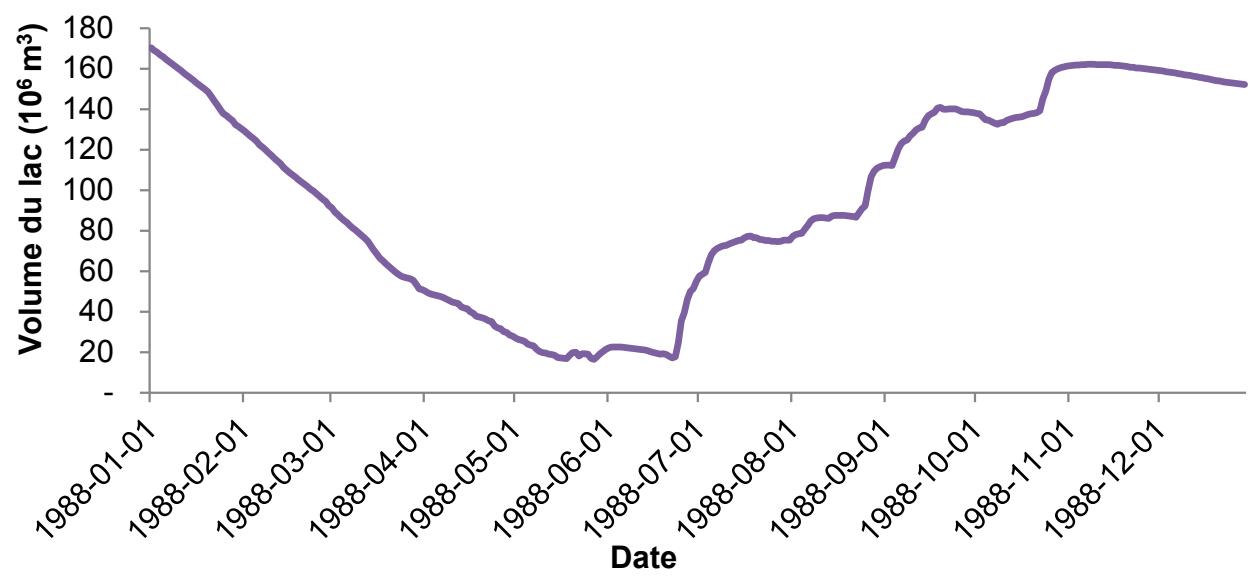

Figure 11. Évolution simulée du volume du lac en 1988. Simulated evolution of the lake volume in 1988.

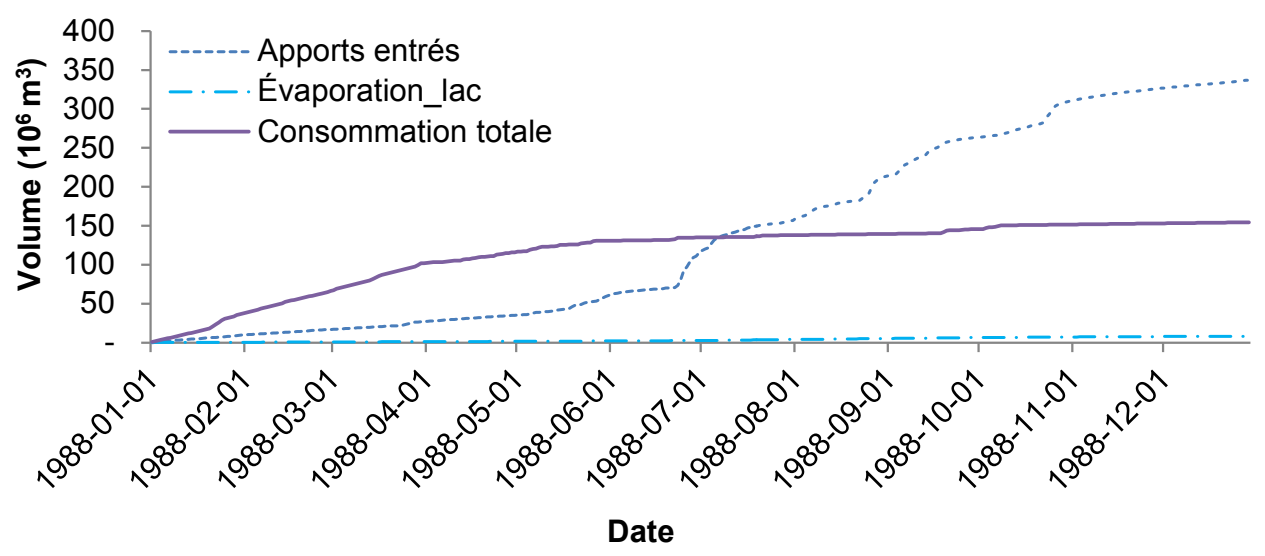

Figure 12. Volumes cumulés entrants et sortants du lac en 1988.

Inflow and outflow cumulative volumes for the lake in 1988.

simulée sur une période de 33 ans (du 01-01-1977 au 31-122009). Pendant cette période, on considère que les demandes autres que celles en irrigation demeurent les mêmes au cours des années. La figure 13 présente la courbe de l'évolution du volume du lac pendant cette période. On constate que le volume minimal du lac reste plus élevé que le volume mort pour 30 des 33 années. Ce qui signifie qu'il était possible, 90,9\% du temps pendant cette période, d'assurer la demande en eau pour l'irrigation. En examinant la courbe d'évolution du volume de la figure 13, on constate que pour les années 1977, 1978 et 1980, il aurait été impossible de satisfaire complètement la demande en eau pour l'irrigation. On constate aussi que le niveau maximal du lac $\left(175 \times 10^{6} \mathrm{~m}^{3}\right)$ n'a pas été atteint pour les années 1977 et 1989. En examinant les données pluviométriques, on constate que ce sont deux années sèches. En effet, en 1977 la pluviométrie annuelle était de $1359 \mathrm{~mm}$ (probabilité au dépassement de $96 \%$ ) et en 1989, elle était de $1533 \mathrm{~mm}$ (probabilité au dépassement de $86 \%$ ).
La figure 14 présente un zoom sur les années 1977 à 1980, afin d'examiner la problématique d'irrigation pour l'année 1977 et 1978. Les figures 15 à 17 présentent, quant à elles, la hauteur d'eau moyenne dans les rizières du bassin versant en relation avec la pluviométrie pour ces mêmes années. Rappelons que le sous-modèle de rizière (NGUYEN et al., 2018) et le sous-modèle de gestion du réservoir considèrent que la hauteur d'eau est la même dans toutes les rizières du bassin versant. Sont également illustrées sur les figures 15, 16 et 17 , les hauteurs minimale et maximale requises dans les rizières en fonction du stade de croissance du riz (voir NGUYEN et al. 2018).

Au début de l'année 1977, on constate (Figure 14) que le lac est plein à ras bord. Ce qui signifie que le volume est satisfaisant pour satisfaire les besoins en irrigation pendant la période sèche. On constate par la suite que la saison des pluies survient en 1977 au mois de juin, plutôt qu'au mois de mai, ce qui a provoqué une insuffisance d'eau dans la phase de préparation du sol et dans la phase végétative du riz (Figure 15). 


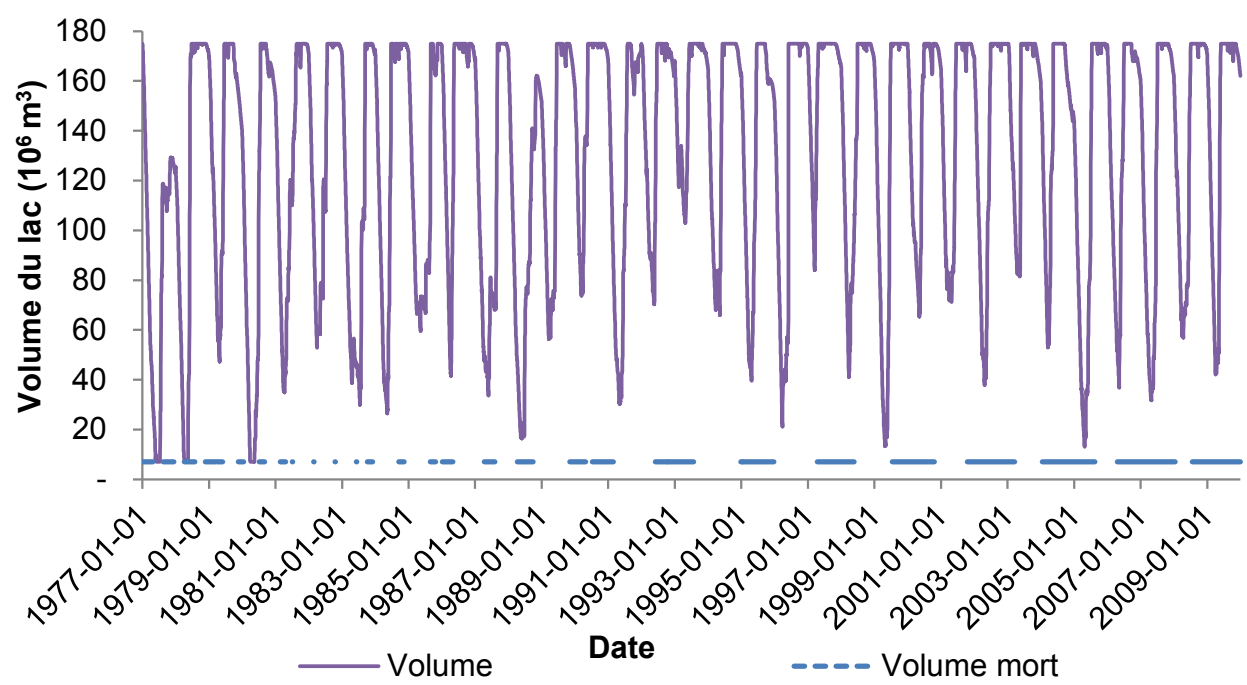

Figure 13. Volume simulé du lac (1977-2009).

Simulated volume of the lake (1977-2009).

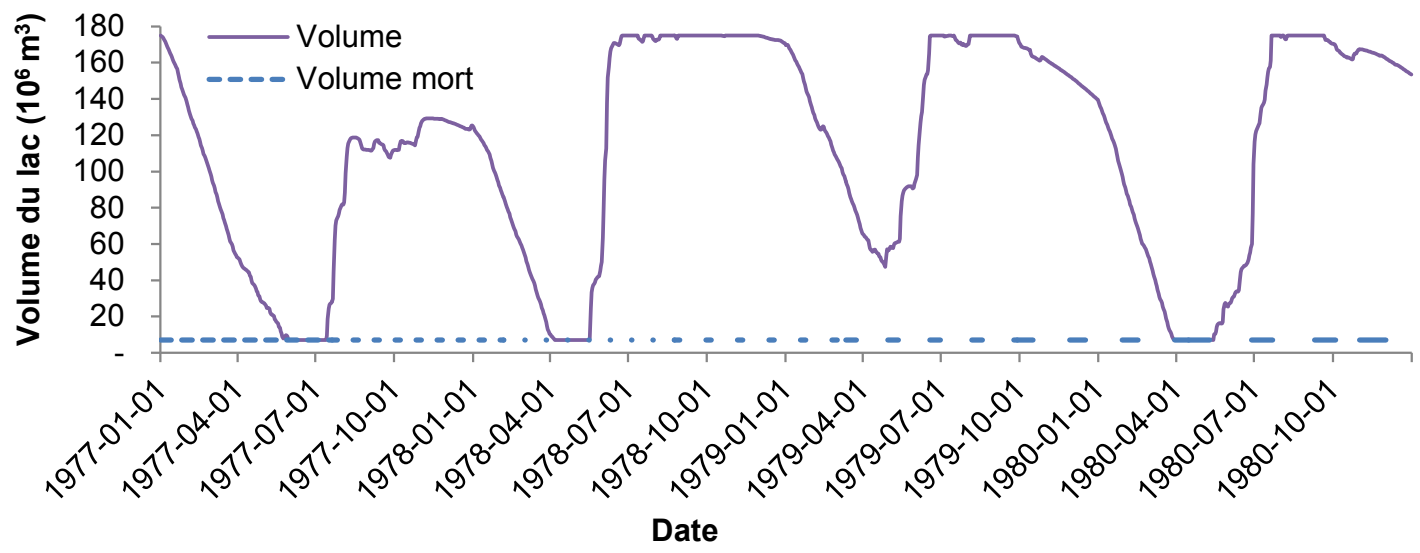

Figure 14. Volume simulé du lac (1977-1980).

Simulated volume of the lake (1977-1980).

En 1978, le volume d'eau stocké en début d'année est d'environ $125 \times 10^{6} \mathrm{~m}^{3}$ (Figure 14). Ce qui provoque l'arrêt de l'irrigation du 28-03-1978 au 01-05-1978 (Figure 16). Au cours de la saison des pluies de l'année 1979, les précipitations n’ont pas été abondantes. Ainsi, au début de l'année 1980, le volume du lac n'est que de $138,4 \times 10^{6} \mathrm{~m}^{3}$ (Figure 14). Les besoins en irrigation n'ont pas pu être satisfaits entre le 13-041980 et le 15-05-1980 (Figure 17), alors que le riz est en pleine croissance.

Sur la base de l'analyse de la capacité du lac à satisfaire aux besoins en irrigation, il est possible d'envisager certaines solutions :

- Dans le cas où le niveau maximal du lac n'est pas atteint en 1978 et en 1989, il aurait fallu diminuer la surface des rizières à irriguer. Ceci montre comment, à partir des résultats de simulation de la gestion du lac, on peut prévoir un plan d'irrigation. Selon le volume stocké en début d'année, il est ainsi possible de planifier une diminution de la surface des rizières à irriguer.

- Dans le cas où le niveau minimal du lac est atteint, il aurait fallu attendre le début de la saison des pluies pour commencer une nouvelle culture.

\section{CONCLUSIONS}

Ce travail a permis de modéliser et de simuler l'hydrologie sur un bassin versant où l'on retrouve un ensemble de problèmes complexes liés à l'irrigation des rizières et à la satisfaction de différents besoins en eau. Cette complexité a conduit au développement de sous-modèles qui, ajoutés au 


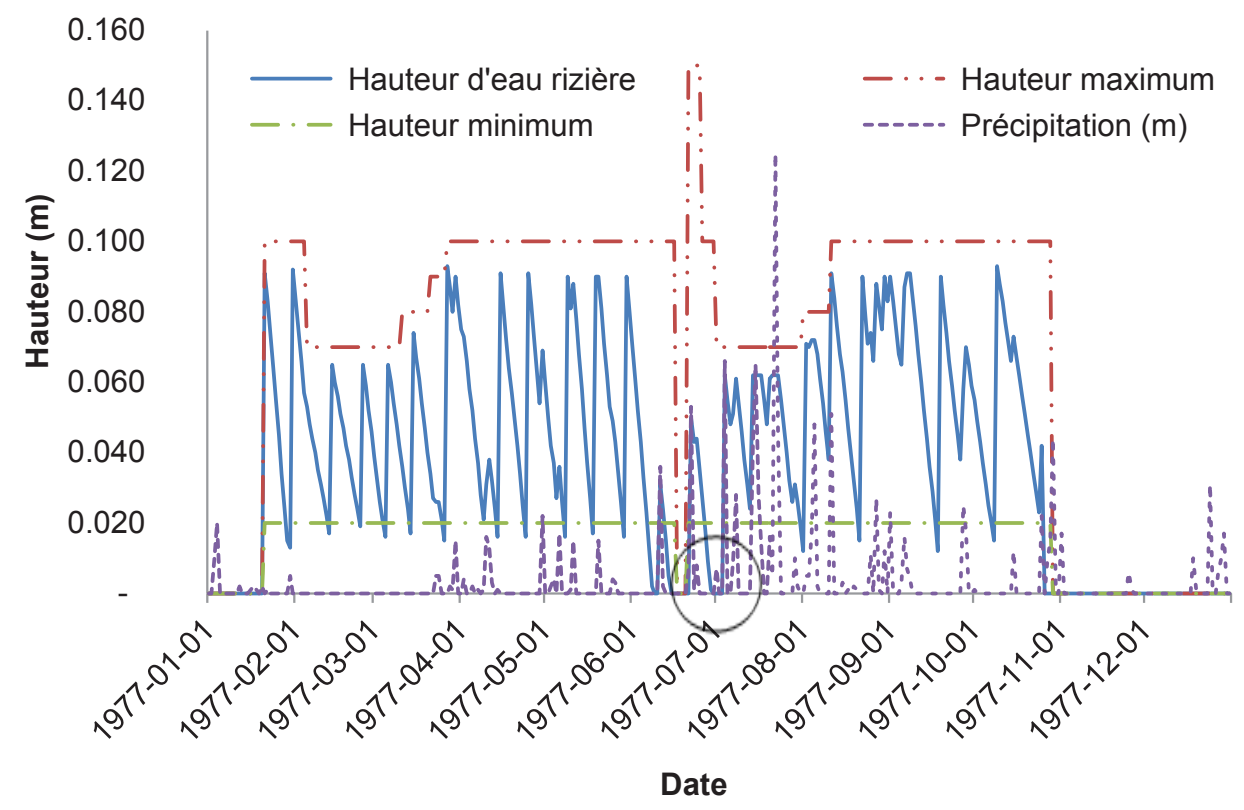

Figure 15. Illustration du problème d'irrigation en 1977 (la saison des pluies débute trop tard). Illustration of the irrigation problem in 1977 (the rainy season begins too late).

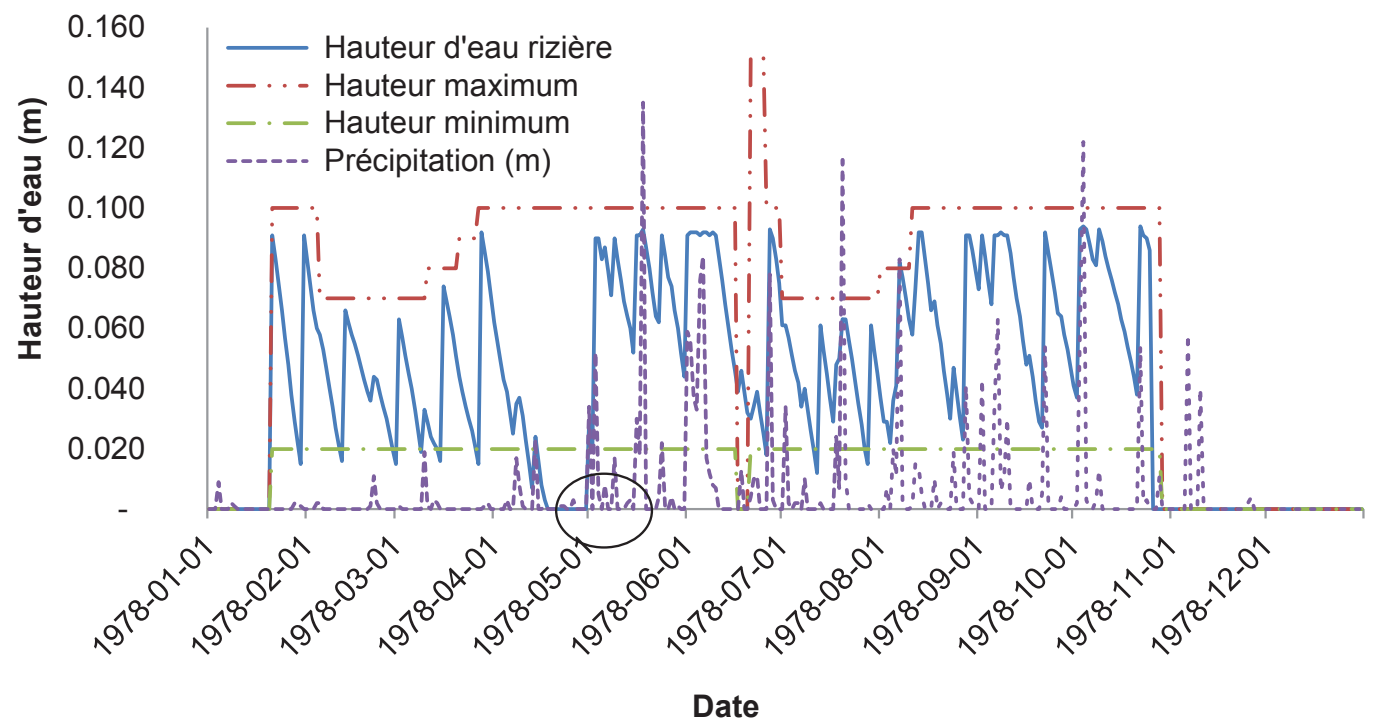

Figure 16. Illustration du problème d'irrigation en 1978 (volume insuffisant dans le lac). Illustration of the irrigation problem in 1978 (insufficient volume in the lake).

modèle Hydrotel, permettent de réaliser la gestion intégrée des ressources en eau du bassin versant de la rivière Cau.

Deux sous-modèles ont dû être développés : un pour la gestion des rizières (NGUYEN, 2016; NGUYEN et al., 2018) et un autre pour la gestion du lac. Le sous-modèle des rizières est un nouvel outil qui permet de déterminer les besoins en irrigation des rizières. Le sous-modèle de gestion du lac est un nouvel outil qui permet de réguler et d'opérer le lac afin de répondre à des objectifs multiples.
L'analyse de la capacité du lac à répondre aux besoins en irrigation des rizières a montré que, sur la période de simulation de 33 ans (1977-2009), il y a trois années (1977, 1978 et 1980) où les réserves en eau du lac n'ont pas pu assurer toute la demande en irrigation. On a constaté aussi que la pluviométrie totale et la date d'arrivée de la saison des pluies influencent fortement la capacité du lac à satisfaire les besoins en eau pour l'irrigation des rizières. Les résultats de simulation permettent de trouver des solutions pour améliorer le service d'irrigation des rizières. 


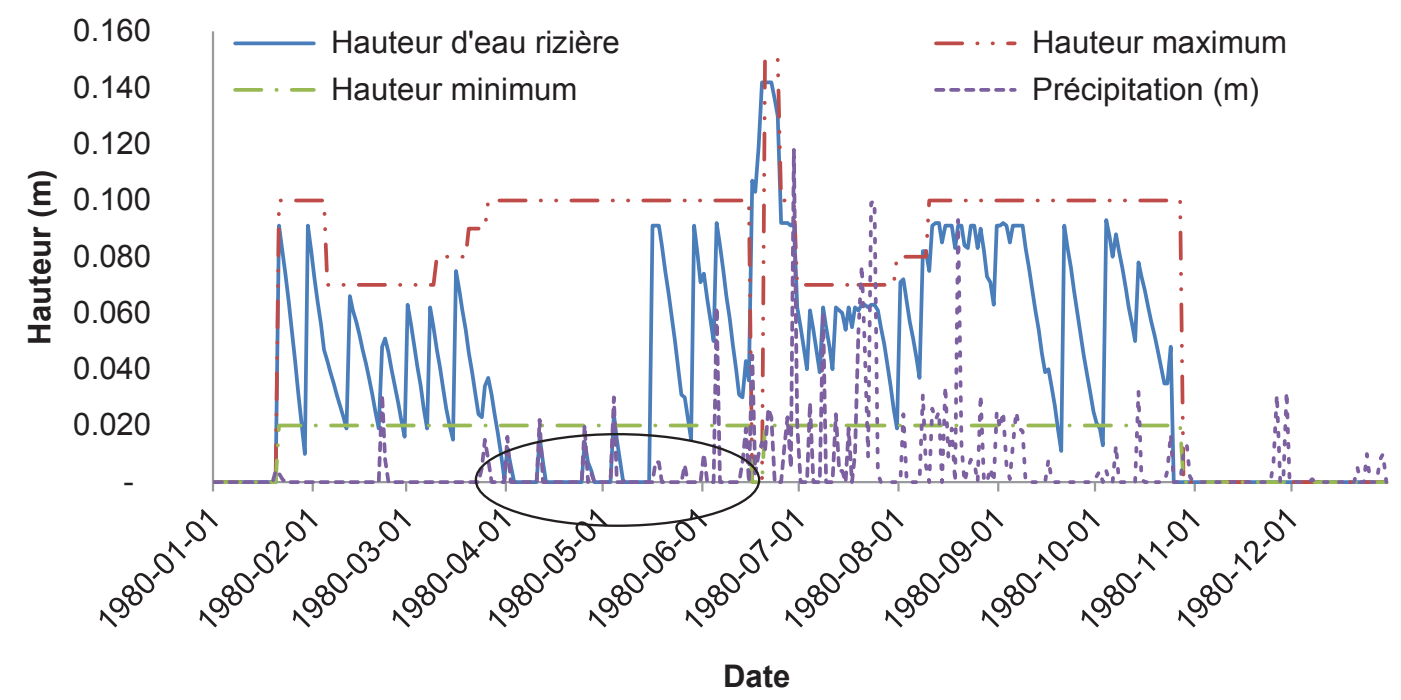

Figure 17 Illustration du problème d'irrigation en 1980 (volume insuffisant dans le lac). Illustration of the irrigation problem in 1980 (insufficient volume in the lake).

En effet, ce travail a permis de proposer des solutions pour améliorer la gestion du lac. En année sèche (comme en 1978 et en 1989), le volume maximal n'étant pas atteint, il faudrait diminuer la surface cultivable des rizières pour assurer une bonne production rizicole. Dans le cas où les pluies arrivent plus tard en saison des pluies, la solution proposée est de retarder le début de la nouvelle culture.

\section{RÉFÉRENCES BIBLIOGRAPHIQUES}

FORTIN J.P., R. MOUSSA, C. BOCQUILLON et J.P. VILLENEUVE (1995). Hydrotel, un modèle hydrologique distribué pouvant bénéficier des données fournies par la télédétection et les systèmes d'information géographique. Rev. Sci. Eau, 8, 97-124.

FORTIN J.P. et A. ROYER (2004). Le modèle hydrologique HYDROTEL - bases théoriques. INRS, Centre Eau Terre Environnement, Québec (QC), Canada, 80 p.

HENK J.M., OGINK H.J.M., N.B. NGUYEN et X.P. NGUYEN (2005). SECO GRONER/ WL, Rapport Delft Hydraulic, Delft, Pays-Bas, 81 p.

LE Q.V. (2011). Critère de conception des ouvrages hydrauliques pour le deltat de la rivière rouge. Ministère de l'Agriculture et Développement rural, Hanoi, Vietnam.

MINISTĖRE DES RESSOURCES EN EAU (MRE) (1988). Critère 'TCVN 4118-85'. Dessins de système des canaux d'irrigation, MRE, Hanoi, Vietnam.
NGUYEN H.T. (2012). Apport de la modélisation hydrologique distribuée à la gestion intégrée par bassin versant des ressources en eau. Thèse de doctorat, Univ. Québec, INRS, Canada, $181 \mathrm{p}$.

NGUYEN X.T. (2016). Modélisation de l'impact des rizières sur le régime d'écoulement de la rivière Cong au Vietnam. Thèse de doctorat, Univ. Québec, INRS, Canada, 181 p.

NGUYEN X.T., S. DUCHESNE, N. KOKUTSE, B. TOUMBOU et J.P. VILLENEUVE (2018). Modélisation de rizières irriguées et implémentation dans HYDROTEL : application au bassin versant de la rivière Cau du Vietnam. Rev. Sci. Eau, 31, 217-233.

ROUSSEAU A.N., A. MAILHOT, R. TURCOTTE, M. DUCHEMIN, C. BLANCHETTE, M. ROUX, N. ETONG, J. DUPONT et J.P. VILLENEUVE (2000). GIBSI - An integrated modeling system prototype for river basin management. Hydrobiologia, 422/423, 465-475.

VILLENEUVE J.P., A.N. ROUSSEAU, A. MAILHOT, E. SALVANO, B. SIMARD, R. CORRIVEAU et S. TREMBLAY (2003). Guide de l'usager de GIBSI (Version II - 2003). Rapport R-549-g2, INRS, Centre Eau Terre Environnement, Québec (QC), Canada, 99 p.

VILLENEUVE J.P., C. BLANCHETTE, M. DUCHEMIN, J.F. GAGNON, A. MAILHOT, A.N. ROUSSEAU, M. ROUX, J.F. TREMBLAY et R. TURCOTTE (1998). Rapport final du projet GIBSI : gestion de l'eau des bassins versants à l'aide d'un système informatisé - Tome 1. Rapport R-462, INRS, Centre Eau Terre Environnement, Québec (QC), $371 \mathrm{p}$. 